\title{
Neurochemical and Behavioural Changes in Rat Models of Depression
}

\author{
Ivan Skelin, ${ }^{\text {a, }, \text {, }}$ Tomislav Kovačević,, a,b,\#, and Mirko Diksic ${ }^{\mathrm{a}, \mathrm{b}, *}$ \\ ${ }^{a}$ Cone Neurosurgical Research Laboratory, Montreal Neurological Institute, Department for Neurology and \\ Neurosurgery, McGill University, H3A2B4 Montreal QC, Canada \\ ${ }^{\mathrm{b}}$ Faculty of Medicine, J. J. Strossmayer University of Osijek, 31000 Osijek, Croatia
}

RECEIVED DECEMBER 18, 2010; REVISED MARCH 11, 2011; ACCEPTED MARCH 11, 2011

\begin{abstract}
Depression is one of the most prevalent psychiatric disorders with significant lifetime prevalence. The study of human depression is hindered by the relatively significant heterogeneity of this disorder. As a result of these heterogeneities, studies using a sound and representative animal model of depression are extremely valuable and, in general, provide important information regarding the neurochemical/biological changes occurring during depression and the subsequent treatment of its symptoms. In this review we concentrate on two solid rat models of depression: olfactory bulbectomized (OBX) rats and Flinders Sensitive Line (FSL) of rats. These two models were selected because they possess all three characteristics of a good animal model; face, predictive and constructive validities. We also emphasize the brain serotonergic system and we discuss the open field and forced swim test to assess behaviour. (doi: $10.5562 /$ cca1817)
\end{abstract}

Keywords: depression, olfactory bulbectomized rat model of depression, Flinders sensitive line rat, Flinders Resistant line rat, animal model of depression

\section{INTRODUCTION}

Depression is a brain disorder which produces major disability. Its aetiology and mechanisms are not yet well understood. According to different reports, the lifetime prevalence of depression ranges between a few percent ${ }^{1}$ to as much as $15-20 \%{ }^{2}$ In addition, depression has not yet been associated with any specific and sensitive structural alterations of the brain, and there is no biomarker of the disorder. Its diagnosis is generally established based on the Diagnostic and Statistical Manual of Mental Disorders (DSM-IV), ${ }^{3}$ but the term "depression" actually represents a number of disorders, including major depressive disorders. The disease is manifested by a loss of interest in pleasure (anhedonia), feelings of guilt, depressed mood, disruption of sleep and/or appetite, low energy, poor concentration and suicidal ideations and attempts. It should be noted that not all of the aforementioned symptoms have to be present in a single patient. Further, depression is a collection of heterogeneous symptoms and neurochemical alterations that result in several hypotheses/theories of the underlying mechanisms. For example, deficiencies in dopaminer$\mathrm{gic}^{4}$ and/or the serotonergic functions ${ }^{5}$ are parts of the so-called monoamine theory of depression. Because anhedonia and loss of motivation are core features of depression, it has been proposed that a disturbance in the brain reward system may be involved in the pathogenesis of major depression. ${ }^{6}$ Also, the aetiology of depression has been correlated to stress, as well as genetic factors. However, no single gene has been discovered, suggesting that the development of depression is a consequence of the complex interplay between multigenic-inherited susceptibility and environment. ${ }^{7}$ The study of depression in the human population is therefore very difficult. Animal models of depression have been used to overcome some of these challenges.

Several review papers have been published on animal models of depression. ${ }^{7-10}$ As is generally the case with many different models of human diseases, animal models show varying levels of similarities to each disease. Accordingly, different animal models have been used to study different aspects of these diseases. Indeed, there have been other animal models of depression, based on behavioural despair, reward insensitivity or stress, which have been discussed recently in other published reviews, ${ }^{8,9}$ and therefore will not be described further here. In a recent review, ${ }^{7}$ it is clearly stated that

$\dagger$ This article belongs to the Special Issue Chemistry of Living Systems devoted to the intersection of chemistry with life.

$\$$ Present address: Department of Neuroscience, The University of Lethbridge, 4401 University Dr. W., Lethbridge, AB T1K 3M4, Canada

\# These authors made an equal contribution to this review.

* Author to whom correspondence should be addressed. (E-mail: Mirko.diksic@mcgill.ca) 
models that may be good predictors of an antidepressant effect (e.g., learned helplessness, the forced swim and tail suspension tests) are nonetheless unrealistic models of human depression, as they show behavioural changes following acute administration, which is not the case in the treatment of human depressive symptoms. Animal models of depression need to fulfill three basic criteria: ${ }^{8}$ 1) face validity (how close the model resembles the psychiatric condition); 2) predictive validity (how close the action of the drugs in the model resembles the action of the drug in humans); and 3) constructive validity (the consistency of the model with the theoretical rationale). According to the literature, ${ }^{8-10}$ there are two very sound rat models of depression: olfactory bulbectomized mice and rats $^{10}$ and the Flinders Sensitive Line of rats. ${ }^{9}$ Accordingly, the present review concentrates on these two models. However, there have been numerous models investigated, but many of them do not possess all three of the above noted characteristics, and, as such, will not be discussed in this review. O'Neil and Moore ${ }^{11}$ wrote: "the OBX test is valuable in that it has been one of the few tests that mimics the slow onset of antidepressant action reported in clinical studies" and concluded that "the convergence of data from markers as diverse as behaviour, neurochemistry, as well as endocrine and immune function, suggest that this model will continue to shed valuable light on depressive mechanisms and treatment for some time to come".

The surgical removal of the olfactory bulbs produces alterations in many neurotransmitter systems and anatomical pathways in the brain, and also produces immunological changes. ${ }^{7,10-13}$ Neuronal changes have been reported in structures deemed to play an important role in affective and cognitive functioning, which are also the target of the olfactory bulb projections, such as the amygdala, greater hippocampal formation, and the bed nucleus of the stria terminalis. ${ }^{10,14}$ The amygdala and hippocampus have important influences on frontal cortical striatal circuitry. ${ }^{15}$ Similarly, dysfunction of the serotonergic and dopaminergic system have been demonstrated in the amygdala of the FSL rats. ${ }^{16,17}$ There is a possibility that chronic sensory deprivation caused by the removal of the rat's most important sensory modality, olfaction, represents a high intensity stressor. However, the animals rendered anosmic with zinc sulphate, although showing a reduced habitation and enhanced reactivity, similar to OBX rats, do not show an increase in basal activity level when tested in the open field arena, unlike the OBX rats, suggesting that the behaviour changes in the OBX rats are independent of anosmia. $^{18}$

The FSL rats were obtained as outbred by selective breeding from the Sprague-Dawley (SPD) rats. ${ }^{19,20}$ It should be noted that the OBX rats are considered to model agitated depression, ${ }^{10,21,22}$ while the FSL rats represent a model of retarded depression. ${ }^{23}$ In recent reviews, these two models have been identified as the best for the evaluation of the biochemical characteristics of the human depressive state. ${ }^{13,23,24}$

It is always questioned which parameters of serotonergic or other neurotransmission one should study in an animal model to obtain the best characterization of a deficiency related to a disease (e.g., depression), as well as the biochemical changes occurring after the elimination of such deficiencies with treatment. There are many parameters that describe, to some extent, serotonergic neurotransmission (e.g., 5-HT synthesis, density and functionality of different 5-HT receptors, 5-HT transporter, and interaction with the dopaminergic and noradrenergic systems), but only a few of them can be studied at any given time. For our discussion, we selected the following parameters: (1) 5-HT synthesis, one of the most important factors of serotonergic neurotransmission; ${ }^{25}$ (2) $5-\mathrm{HT}_{1 \mathrm{~A}}$ receptors, shown to be important in 5-HT synthesis control. ${ }^{26}$ In addition, the densities of these sites are different between the OBX and sham operated rats, ${ }^{27,28}$ as well as between the FSL and control FRL rats; ${ }^{29}$ (3) $5-\mathrm{HT}_{2 \mathrm{~A}}$ receptors, which exhibit differences between depressed and normal subjects ${ }^{30,31}$ and appear to be affected by the OBX surgery. ${ }^{28,32}$ Further, binding to the $5-\mathrm{HT}_{2 \mathrm{~A}}$ receptors is decreased following paroxetine and desipramine treatments in subjects with major depression; ${ }^{33,34}$ (4) Cholinergic, noradrenergic, dopaminergic, and GABA-ergic alterations in the FSL rats ${ }^{35-38}$ and (5) Alteration in second messenger signalling. ${ }^{39}$ Studies have also shown that $5-\mathrm{HT}_{1 \mathrm{~A}}$ receptors are supersensitive in the FSL rats when compared to the SPD controls. ${ }^{16,17}$ Further, 5-HT synthesis has been identified as a parameter that is affected by $\mathrm{OBX}^{40,41}$ and is normalized by antidepressant treatments. ${ }^{27,41}$

As noted, we concentrate on the OBX and FSL rat models of depression in this review, because our group has acquired a substantial volume of data on the serotonergic system in these two models, and that data is not available on any other animal model. In addition, these two models have been described by others ${ }^{4,10}$ as the most realistic animal models of depression. The majority of the discussion will concentrate on the brain serotonergic system, but other deficiencies and possible parallels with human depression will also be discussed.

\section{Olfactory Bulbectomized Rat as a Model of Depression}

The removal of the olfactory bulbs induces neurochemical, neuroanatomical, physiological, endocrine and behavioural changes in rats, which are similar to those present in human depression. ${ }^{10}$ It was shown, more than three decades ago, that behavioural changes in olfactory bulbectomized (OBX) rats (e.g., hyperactivity, passive avoidance and irritability) were reversed following chronic antidepressant treatment. ${ }^{42}$ The relationship 
between the length of antidepressant treatment and time-onset of behavioural normalization in OBX rats accords with the findings in depressed patients (it typically takes a minimum of two weeks for a drug to induce a clinical improvement). This characteristic contributes to the face validity of OBX as a model of depression. ${ }^{43}$ From that point onwards, OBX was used as a model to predict antidepressant characteristics of putative antidepressant compounds. The utilization of this model in biomedical research has now been extended to understand many biological changes that are comparable with human depression. ${ }^{44}$ In addition, endocrine changes also present, to some extent, in human depression (e.g., impaired hypothalamic pituitary-adrenal axis $^{10,45,46}$ ) were observed in the OBX rats, as was hyperphagia with no weight gain, ${ }^{46,47}$ and altered immune functioning and circadian pattern. ${ }^{10,44}$ Recent research showed that physiological changes, such as higher core body temperature and decreased heart rate variability, occurred 3-5 days post surgery and persisted for a relatively long time, possibly permanently. ${ }^{48}$

\section{Surgical Procedure and its Subsequent Neuro- Anatomical Implications}

The surgical procedure of olfactory bulb (OB) removal in rats has been previously described in great detail. ${ }^{40,49}$ In brief, Sprague-Dawley (SPD) rats (weighting 200-250 g) are anaesthetized with inhalatory anaesthetics, isoflurane or halothane, and the small hole is opened on the frontal bone, $5.2 \mathrm{~mm}$ anterior from the bregma and approximately $2 \mathrm{~mm}$ on both sides from the midline. The olfactory bulbs are visualized with a surgical microscope, cut and aspirated with a dull needle attached to a vacuum water pump, taking care not to damage the frontal lobes. The hole is filled with a hemostatic sponge to prevent bleeding and the skin is closed with sutures. The control sham-operated rats are operated in the same way, except that the olfactory bulbs are not removed. A period of two weeks is sufficient for the bulbectomy syndrome to develop. A recently described technique of photochemically induced bulbectomy offers a new possibility in inducing OBX syndrome in rats. ${ }^{50}$

Olfactory bulbectomy-induced changes, in many brain regions, are the result of direct or indirect connectivity of those regions with olfactory bulbs. ${ }^{24,40}$ Subsequent extensive changes were referred to retrograde, anterograde and transneuronal degeneration, including the damages that are a result of vascular blood supply deprivation. ${ }^{24}$ In addition to the bulbectomy-associated axotomy of 5-HT fibres, the reorganization of 5-HT projections to the forebrain is continued by collateral sprouting and synaptogenesis. ${ }^{46}$ Furthermore, neuronal degeneration occurs in many accessory projection areas (e.g., cortex, hippocampus, amygdala, locus coeruleus and raphe nuclei). Structural changes in the locus coeruleus and raphe nuclei likely underlie the noradrenergic and serotonergic imbalances observed in these rats. ${ }^{24}$ Cortico-hypocampal-amygdala circuit changes were reported in humans suffering from depression. ${ }^{51}$ Magnetic resonance imaging studies have reported changes in signal intensities in the cortex, hippocampus, caudate and amygdala of OBX rats. ${ }^{52}$ The reduction noted in the hippocampal neurogenesis in the OBX rats was reversed by a 28 day treatment with citalopram which increased the proliferation of the neuronal progenitors and accelerated the differentiation of the progenitors into the adult granule neurons in the dentate gyrus. That effect was not found in the sham operated animals. ${ }^{53}$ Others reported a decrease in hippocampal volume which correlates with an increase in the ventricular cisterns of the OBX rats. ${ }^{24}$ Changes in the size of the ventricular volume were correlated with the degree of cognitive impairment and memory. ${ }^{24,54}$ Similarly, Song and Leonard ${ }^{24}$ reported changes within the piriform cortex with a reduction in the number of synapses and dendritic spines and shafts in the OBX rats. These latter reductions were reversed by chronic antidepressant treatment. ${ }^{24} \mathrm{OBX}$ rats have a reduced number of tryptophan hydroxylase (TPH)-positive cells in the raphe nuclei. ${ }^{55}$

\section{Behavioural Changes in OBX Rats}

Olfactory bulbectomized (OBX) rats show an alteration in normal behaviour. ${ }^{10}$ Increased activity in an open field test is one of the quickest and most consistently reported behavioural changes in the OBX rats, ${ }^{10,49,56}$ which does not occur as a consequence of the loss of smell. $^{42}$ The lesioning of the serotonergic projections to the olfactory bulb lesion (OB) with a local injection of the neurotoxin 5,7-dihydroxytrypramine (5,7-DHT) also produced hyperactivity in the open field test, which was also the case in OBX rats, ${ }^{10,24}$ suggesting effects of the brain serotonergic system on the behavioural changes observed in the OBX rats. The hyperactivity was reversed by chronic administration of amitryiptyline and mianserine. OBX rats showed a deficit in learning to avoid the foot shock by remaining on a platform that administers electrical shocks longer than the control group (passive avoidance test). ${ }^{10,42}$ The neuronal pathways from the $\mathrm{OB}$ project directly to the anterior cortical nucleus of the amygdale. The amygdala modulates memory processing in other brain regions. Therefore, alterations in amygdala functioning are thought to be responsible for altered memory processing following OBX surgery. Treatment with the cholinesterase inhibitor, physostigmine, and the selective muscarinic (M1) agonist, oxotremorine, improved learning and memory related behaviour. Furthermore, the choline acetyltransferase (ChAT) fluorescence was decreased in the hippocampus, amygdala and cortex of OBX rats. ${ }^{57}$ 


\section{Serotonergic System Alterations in OBX Rats}

The first measurement of regional 5-HT synthesis in the OBX rats using the $\alpha-\left[{ }^{14} \mathrm{C}\right]$ methyl-L-tryptophan ( $\alpha$-MTrp) autoradiographic method was reported in $2003 .{ }^{40}$ The method was previously described, ${ }^{58,59}$ and the details will not be repeated here. Watanabe et al. ${ }^{40}$ found increased 5-HT synthesis in the majority of the 5-HT projection regions in OBX rats, with a synthesis reduction in the raphe nuclei, the location of the 5-HT neuron cell bodies. The reduction in the synthesis, in part, possibly relates to the loss of the cells in the raphe following OBX surgery. ${ }^{60}$ However, the reduction in 5-HT synthesis could also be related to a direct inhibition of 5-HT synthesizing enzyme tryptophan hydroxylase (TPH) with the end product, 5-HT, via feedback through the $5-\mathrm{HT}_{1 \mathrm{~A}}$ autoreceptors. ${ }^{40} \mathrm{~A}$ chronic treatment with citalopram (an SSRI) reduces the 5-HT synthesis rate in the OBX rats and brings it to a level comparable with the control; sham-citalopram and sham-saline rats. ${ }^{41}$ The 5 -HT synthesis rate in the OBX rats treated with citalopram was lower in only a few regions relative to the sham-saline rats. This is explained by the combined action of higher extracellular levels of 5-HT in OBX rats treated with citalopram that acts through the autoreceptors $\left(5-\mathrm{HT}_{1 \mathrm{~A}}\right.$ has an inhibitory function on 5-HT synthesis) that were not fully desensitized in OBX rats compared to the sham rats. ${ }^{41}$ When, in OBX and sham operated rats, an acute pindolol $\left(5-\mathrm{HT}_{1 \mathrm{~A} / \mathrm{B}}\right.$ antagonist) treatment was combined with an acute citalopram treatment, 5-HT synthesis in the OBX rats remained high in the terminal regions. ${ }^{61}$ This may be explained by the higher responsiveness of the $5-\mathrm{HT}_{1 \mathrm{~A}}$ receptors in the OBX rats. As we previously discussed, chronic treatment with citalopram (and other antidepressants) does not produce behavioural changes in sham operated rats. This correlates to neurochemical findings of 5-HT synthesis. ${ }^{41}$

The 5-HT transporter (SERT) studies revealed significantly higher SERT densities across many brain regions, ${ }^{28}$ when the OBX rats were compared to the sham rats. In accordance with this finding, it was reported that the OBX rats have a higher SERT density and higher level of the tryptophan hydroxylase enzyme in the frontal cortex, suggesting the 5-HT hyperinnervation of that region. ${ }^{46,62}$ The elevation of the SERT in the OBX rats could be related to the proliferation of the glia after the neuronal cells die. ${ }^{63}$

Cerebral glucose utilization (CGU) represents total brain energy usage and it was found that there is a greater CGU in the terminals than in the cell bodies. ${ }^{64}$ However, the CGU in the OBX rats was found to be lower than in the sham controls. This could be due to the increased levels of the inhibitory neurotransmitters (glycine), and the decreased levels of the excitatory neurotransmitters (glutamate, aspartate). Other causes could be increased 5-HT synthesis and tissue content, considering the mostly inhibitory action of 5-HT on target neurons and/or a non-specific result of a brain injury. ${ }^{65} \mathrm{~A}$ micro-dialysis study showed reduced extracellular 5-HT levels in the dorsal hippocampus and basolateral amygdala in rats following OBX surgery. ${ }^{66}$ 5-HT turnover in OBX rats was elevated in all brain regions investigated, ${ }^{66}$ suggesting the elevation of 5-HT synthesis which accords with the autoradiographic measurements. $^{40,41}$ The administration of D-fenfluramine (a 5-HT releasing and reuptake blocking drug) and the SSRI, fluvoxamine, induced an attenuated increase in 5HT levels in the OBX rats. ${ }^{66}$ Accordingly, this elevation in 5-HT would support increased 5-HT synthesis rates $^{40,41}$ and could be the result of an altered control mechanism of 5-HT exocytosis. Decreased microdyalisis values of 5-HT only accounts for the extracellular 5-HT, rather than the intracellular.

Chronic citalopram treatment produced a novel CGU (cerebral glucose utilization) pattern, rather than a normalized CGU pattern, when compared to the sham group. ${ }^{67}$ The administration of D-fenfluramine reduces the rCGU (regional cerebral glucose utilization) in the sham, but not the OBX, rats. This finding suggests a decreased reactivity of the 5-HT system in OBX rats on the fenfluramine challenge. This could be a result of the presynaptic (reduced synthesis storage and release, or an increased 5-HT clearance) or postsynaptic receptor (decrease in density, affinity or sensitivity) changes that occur in the OBX rats. ${ }^{68}$ As previously mentioned, the 5-HT effect on neuronal activity is mostly inhibitory. It has been reported that D-fenfluramine blocks the induction of the long term potentiation (LTP) which correlates with a reduction in the parameters for glucose utilization. ${ }^{69}$

Watanabe and colleagues reported that chronic treatment (14 day) with $20 \mathrm{mg} / \mathrm{kg} / \mathrm{day}$, as opposed to $10 \mathrm{mg} / \mathrm{kg} / \mathrm{day}$, of chronic treatment (14 day) with buspirone $\left(5-\mathrm{HT}_{1 \mathrm{~A}}\right.$ presynaptic agonist, postsynaptic $\mathrm{D} 2$ antagonist and adrenergic alpha2 antagonist) decreased 5-HT synthesis, as measured using $\alpha$-MTrp autoradiography. This decrease and normalization of regional 5-HT synthesis coincides with the normalization of open field hyperactivity, ${ }^{27}$ which was considered to represent the antidepressant-equivalent action of buspirone. A reduction in the synthesis following buspirone treatment ${ }^{70}$ was found in many cortical regions (e.g., cingulated, frontal, parietal), but not in the ventral tegmental area (VTA), DR, MR or superior colliculi (SC). This suggests that the $5-\mathrm{HT}_{1 \mathrm{~A}}$ and $5-\mathrm{HT}_{1 \mathrm{~B}}$ receptor sensitivities may be regionally specific. Furthermore, the sham rats did not show a reduction in 5-HT synthesis, presumably because of receptor desensitization. Others reported a dysfunction of $5-\mathrm{HT}_{1 \mathrm{~A}}$ receptors in depressed individuals, which is consistent with the findings of 
$5-\mathrm{HT}_{1 \mathrm{~A}}$ receptor alteration in the $\mathrm{OBX}$ rats. ${ }^{70}$ The density of the $5-\mathrm{HT}_{1 \mathrm{~A}}$ receptors in the OBX rats was found to be lower than in the sham controls. This finding could be explained by the elevated tissue 5-HT in OBX animals, which produces a desensitization and receptor internalization leading to an inhibition of 5-HT synthesis by treatment with a $5-\mathrm{HT}_{1 \mathrm{~A}}$ agonist. An antidepressant treatment $(20 \mathrm{mg} / \mathrm{kg} /$ day for 14 days $)$ did not produce a decrease in the $5-\mathrm{HT}_{1 \mathrm{~A}}$ receptor density, but rather a fine tuning in some of the limbic brain areas. On the other hand, the sham rats showed a dose dependant decrease in $5-\mathrm{HT}_{1 \mathrm{~A}}$ receptor density following chronic buspirone treatment. That occurred likely because of the desensitization (down regulation) effect of buspirone on receptor sites. ${ }^{27}$ However, changes in $5-\mathrm{HT}_{1 \mathrm{~A}}$ receptor density, per se, will not be sufficient to explain the drug action in depression. It was found that there is an increase in $5-\mathrm{HT}_{1 \mathrm{~B}}$ and $5-\mathrm{HT}_{2 \mathrm{~A}}$ receptor densities in OBX rats when compared to the sham rats following chronic buspirone ${ }^{28}$ administrations. A decrease in 5-HT transporter densities occurred in both groups.

\section{Noradrenalin System Alterations in OBX Rats}

Several studies showed that noradrenalin brain tissue levels were reduced in OBX rats. ${ }^{24,49}$ Changes in the adrenoreceptor function in human depression are similar to those in OBX rats. ${ }^{24,71}$ As previously reported, desipramine (tricyclic antidepressant) and sertraline (SSRI) reversed low levels of noradrenalin in OBX rats, ${ }^{24}$ confirming its the face validity of the OBX model. An increase in the adrenoreceptors density in the frontal cortex (alpha-2) and in the peripheral blood lymphocytes (beta) has been reported. ${ }^{24} \mathrm{~A}$ clonidine induced hypothermic response, which was reduced in the OBX rats, and a growth hormone to clonidine challenge confirmed reduced function of these receptors in OBX rats. ${ }^{24}$

\section{Dopaminergic System Alterations in OBX Rats}

The deregulation of the dopaminergic system in depression suggests an important role of this system in depression. The behavioural effects of OBX resemble psychomotor agitation (e.g., "agitation-like" behaviour). This observation has led to the characterization of the OBX rats as a model of "agitated" depression. ${ }^{22}$ As measured by micro-dyalisis, OBX rats have higher extracellular levels of dopamine (DA) in the striatum than the sham operated rats, which is likely related to the hyperactivity observed in the OBX rats. ${ }^{72}$ These regional specific differences in the basal levels of dopamine are probably related to the region specific differences in the densities of the dopamine transporter (DAT), a major regulator of dopamine neurotransmis- sion in the brain, as well as in behavioural changes of OBX rats. Some studies suggest a rather prolonged disruption in the dopaminergic neurotransmission in the OBX rats (2004). Similarly, Lumia et al. $^{22}$ found a reduction in dopamine turnover (HVA/DA ratio; homovanillic acid) in the striatum of OBX rats. It has been reported that OBX increases DA D1 and D2 receptor expression and DA mediated adenylate cyclase activity in the olfactory tubercle (OT). ${ }^{73}$ DA has also been implicated in antidepressant treatments, including electro convulsive shock therapy (ECS). Acute ECS increases striatal concentrations of DOPAC [dihydroxyphenylacetic acid] and HVA, but chronic ECS

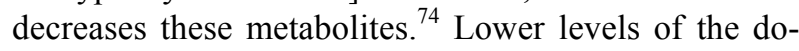
pamine metabolite, HVA, are found in the cerebrospinal fluid of depressed suicide attempters. ${ }^{75}$ High levels of DAT are found on dopaminergic cells in the substantia nigra and ventral tegmental area (VTA), as well as in the projection areas of the basal ganglia using immunocytochemistry. ${ }^{76}$

\section{Glutamate System Alteration in OBX Rats}

Glutamate is abundant in the brain. ${ }^{77}$ It plays an important role in both the physiology and pathophysiology of the central nervous system. Glutamate acts by stimulation ionotropic and metabotropic glutamate receptors. ${ }^{78}$ The involvement of group I mGlu receptors (mGlu1 and mGlu5) in psychiatric disorders such as depression and anxiety has been proposed. Olfactory bulbectomy induces an increase in mGluRla-immunoreactivity (IR), which can be abolished through amitriptyline treatment in the hippocampus. ${ }^{79}$ Similarly, the removal of the olfactory bulbs produces a decrease in the level of mGlu2/3 receptors in the hippocampus, which is reversed by amitriptyline administration. The increase in striatal glutamate release in the OBX rats in response to novelty exposure (e.g., bright field in the open field test) has been associated with glutamate release following stress exposure. ${ }^{80}$ This enhanced glutamate release in the striatum of OBX rats after novelty stress could be related to elevation of plasma corticosterone by $\mathrm{OB}$, because it has been shown that corticosterone affects stress-induced glutamate levels in the brain ${ }^{81}$ and significantly higher basal plasma corticosterone levels are found in OBX rats when compared to the sham-operated controls. ${ }^{82}$ It has been reported that the mGluR5 antagonists, 2-methyl-6-(phenylethynyl)-pyridine (MPEP) and 3-[(2-methyl-1,3-thiazol-4-yl)ethynyl]-pyridine (MTEP), exhibit antidepressant activity in the OBX rats. ${ }^{83,84}$ Riluzole, a drug currently used for slowing the progression of amyotrophic lateral sclerosis (ALS), reduced the hyperemotional responses in OBX rats and significantly lowered the glutamate levels in the frontal cortex after a single injection. ${ }^{85}$ This drug decreases glutamate release and increases glutamate uptake. It also enhances the 
expression of brain derived neurotrophic factor (BDNF) ${ }^{85}$ Because of the exhibited effects, this drug is considered as a potential new antidepressant, with the glutamatergic system playing a role in mood disorders. The $N$-methyl-D-aspartate glutamate ionotropic receptor (NMDA) has been referred to as a locus of action for antidepressant medication and its reduced number in the cortex and amygdala in OBX rats has been reported. ${ }^{86,87}$ Treatment by an NMDA agonist improved learning and memory capabilities of OBX rats. ${ }^{88}$

\section{Acetylcholine System Alteration in OBX Rats}

There is some evidence for a link between depression and nicotine acetylcholine receptors (nAChRs). ${ }^{89}$ The antidepressant actions of nicotine and the targeting of nAChRs by monoamine re-uptake inhibitors suggest a potential role of nAChRs in the aetiology and/or symptomatology of depression. The OBX rats exhibit significantly lower acetylcholine levels in the cortex than intact and sham operated controls or intact rats. ${ }^{90} \mathrm{Ol}-$ factory injury causes retrograde degeneration of cholinergic fibers in OBX rats. That results in decreased cholinergic innervations of the cortex and hippocampus. ${ }^{91}$ Impairment in learning and memory in OBX rats was related to a deficient cholinergic system. Choline acetyltransferase (ChAT) fluorescence was found to be reduced in the cortex, hippocampus and amygdala of OBX rats, but nobiletin (a Citrus Flavonoid) treatment rescues OBX-induced cholinergic neurodegeneration and is accompanied by improvement in impaired memory in OBX mice. ${ }^{92}$ Similarly, it was reported that the administration of $\mathrm{M}_{1}$ postsynaptic agonists and $\mathrm{M}_{2}$ presynaptic antagonists increases performance of OBX rats in memory and learning. ${ }^{57}$ It has also been shown that muscarinic receptor densities were reduced in the amygdaloid cortex, the basal ganglia, hippocampus, hypothalamus, cortex and olfactory regions in OBX rats. ${ }^{32}$ Chronic mianserine and desipramine treatment increased the muscarinic receptor density in OBX rats. ${ }^{32,93} \mathrm{OBX}$ rats are also less sensitive to the induction of seizures following pilocarpine administration. ${ }^{94}$ The release of acetylcholine from the hippocampus is inhibited by 5 -HT trough the $5-\mathrm{HT}_{2}$ receptors. ${ }^{95} \mathrm{Be}-$ cause of its cholinergic alterations, the OBX rats are also used as a model of Alzheimer's disease, as there is an elevation of beta-amyloid in the rat brain after bulbectomy. ${ }^{96}$

\section{Other Alterations in OBX Rats with Possible, Novel Therapeutic Approaches}

Some of the recent results suggest that other systems that are altered in OBX rats may serve as possible biological markers of depression (e.g., genes, neuropeptides). Neuropeptide Y, which was linked to depres- sion, ${ }^{97}$ was shown to be increased in the amygdala of OBX rats. ${ }^{98}$ It has been proposed that oxidative stress is associated with neuronal death and depression. ${ }^{99,100}$ Newer research shows that transcranial magnetic stimulation protects cells from oxidative processes in OBX rats and serves as a possible method of antidepressant treatment. One of the possible explanations of depression is insufficient production of neurotrophic factors (e.g., BDNF) in depressed individuals. ${ }^{101,102}$ Another study has shown that postmortem brains of patients that were exposed to antidepressant medication during their lifetime had higher hippocampal BDNF levels, relative to the post-mortem brains of medication-free normal subjects. ${ }^{103}$ Inconsistent with the findings in depressed humans, OBX rats have increased serum BDNF levels. ${ }^{102}$ A study by Vieyra-Reyes and her colleagues ${ }^{104}$ reviewed the assertion that the cessation of smoking precipitates depressive symptoms. They also showed that olfactory bulbectomy cannot produce depressivelike symptoms in all strains of rats (e.g., no symptoms were developed in the Long Evans rats). Furthermore, chronic nicotine intake displayed antidepressant properties in rodents. They reported an increase of free consumption of nicotine in OBX animals. ${ }^{104}$

To further understand the complexity of the OBX syndrome and its repercussion on neurochemical brain systems in the rat brain, it is important to note the involvement of the opioid system in the development of the OBX syndrome. Subchronic administration of the opioid agonist, SNC80, in OBX rats, reduced hyperemotional responses (struggle, fight, attack, and startle), as well as time spent in an open arm maze. These effects were not observed in the sham rats. Subchronic treatment with the same compound increased levels of 5-HT and its metabolite, 5-hydroxyindol acetic acid (5HIAA), in the frontal cortex, hypothalamus and amygdala in the OBX rats. OBX rats have a reduced number of tryptophan hydroxylase cells in the raphe nuclei. The administration of SNC80 reversed the OBX-induced serotonergic abnormalities in many brain areas. ${ }^{105}$

These findings highlight the need for further research to better understand the differences and complexity of human depression as a heterogenic, rather than a homogenic, brain disorder.

\section{Flinders Sensitive Line}

Flinders Sensitive Line (FSL) rat is an animal model of depression. It has been selectively bred from the Sprague-Dawley strain, based on its supersensitivity to the acute hypothermic effect of the cholinesterase inhibitor, diisopropyl fluorophosphate (DFP). ${ }^{19,20}$ The Flinders Resistant Line (FRL) was also selectively bred from the SPD rats to develop a strain exhibiting a cholinergic sub-sensitivity. The original purpose of the breeding program that resulted in the development of the FSL 
and FRL strains was to develop the rat model of cholinergic resistance. ${ }^{19,20}$ However, the cholinergic sensitivity of FRL rats is not different from the SPD rats. ${ }^{23}$ Following the subsequent development of the cholinergic super-sensitivity theory of depression in humans, ${ }^{106}$ it was suggested that FSL rats may be a useful rat model of depression. The FRL rats are most often used as the normal control strain in the studies of FSL rats. ${ }^{23}$

\section{Behavioural Characteristics of the FSL Model}

From a behavioural perspective, the FSL rats exhibit hypoactivity in the forced swim test, ${ }^{107}$ a widely used screening method for animal models of depression. ${ }^{108} \mathrm{It}$ has been suggested that hypoactivity in the forced swim test is a reflection of behavioural despair, pointing out that the FSL rats may resemble this core feature of human depression (The Diagnostic and Statistical Manual of Mental Disorders-IV; DSM-IV). ${ }^{3}$ FSL rats are also hypoactive in the open field arena, ${ }^{19}$ which could be attributed to psychomotor retardation, but also to novelty-induced anxiety, which suppresses natural exploratory behaviour. ${ }^{109} \mathrm{FSL}$ rats had a decreased interaction with the con-specifics in the social interaction test, ${ }^{35}$ although they did not show anxious behaviour in the non-social anxiety test (elevated plus maze) ${ }^{110}$ Anhedonia (reduced ability to experience pleasure) is one of the key features of depression in humans (DSM-IV) ${ }^{3}$. Normal animals show a preference for saccharine-sweetened solution, relative to water. The absence of this preference is considered to be a sign of anhedonia in animal models. ${ }^{111}$ FSL rats have shown an increased preference for the sweetened solution, relative to the water, in the basal conditions, ${ }^{107}$ suggesting a normal hedonic response. However, following exposure to acute or chronic stress, the preference for the saccharine-sweetened solution in the FSL rats was not maintained, while stress had no effect on the sucrose preference in the control FRL rats. ${ }^{107} \mathrm{FSL}$ rats have shown the normal threshold for intracranial self-stimulation (ICSS), another paradigm testing sensitivity to pleasure. ${ }^{112}$ It is not clear whether the sub-sensitivity of the FSL rats to the induction of cocaine-seeking ${ }^{113}$ is due to the elevated hedonic threshold or other reasons. Therefore, FSL rats have very solid face validity (i.e., resemblance of their behavioural phenotype to depressed humans). Differences in learning abilities have been found in FSL rats, though the direction of the difference depends on the tested parameter. The acquisition of the learned active avoidance response is significantly slower, compared to the FRL rats, ${ }^{23}$ while the acquisition of passive avoidance is preserved. ${ }^{20}$ These findings only partially resemble the consistent findings of cognitive impairment in depressed individuals (reviewed by Austin et al. ${ }^{114}$ ).

\section{Physiological Characteristics of the FSL Model of Depression}

At the physiological level, FSL rats weigh less and have lower appetite than the age-matched FRL rats. ${ }^{23}$ Weight loss is a common symptom of major depressive disorder, although some depressive patients show no weight loss or even weight gain during depressive episodes (DSM-IV). ${ }^{3}$ Another physiological characteristic of the FSL strain that is similar to human depression is sleep disruption. Specifically, the rapid eye movement (REM) sleep phase has an earlier onset than the FRL rats. The percentage of the duration of REM phases, relative to total sleep length, is also higher in FSL rats, ${ }^{115,116}$ resembling the findings in the subset of depressed humans. ${ }^{117}$ The disruption of sleep architecture in FSL rats is not surprising, considering the cholinergic super-sensitivity of FSL rats and the breadth of evidence implicating the role of the cholinergic system in the initiation and maintenance of REM sleep (reviewed by Shiromani et al. ${ }^{116}$ ). A number of immune system alterations consistent with those found in depressed patients have been reported in the FSL rats, such as impaired type 1 immune reaction. ${ }^{118}$

\section{Predictive Validity of the FSL Model of Depression}

The predictive validity of the animal model of psychiatric disease relates to the normalization of aberrant behavioural characteristics of the model achieved exclusively using the drugs known to have clinical efficacy in human populations suffering from the modelled illness. ${ }^{119}$ Different behavioural features of the FSL model, immobility in the forced swim being the most often tested, have been either normalized or at least affected (sildenafi1 ${ }^{120}$ ) following chronic, but not acute, treatment with tricyclic antidepressants (imipramine; ${ }^{107}$ clomipramine ${ }^{121}$ ), selective serotonin reuptake inhibitors (SSRIs) (paroxetine; ${ }^{122}$ fluoxetine ${ }^{35}$ ), cholinergic agonists (nicotine ${ }^{123}$ ), beta3 adrenoceptor agonists (amibegron $^{124}$ ), melatonin receptor agonists (S 20304 ${ }^{125}$ ), corticotropin releasing factor (CRF) antagonists (CP154, $523^{126}$ ), neurotrophin (neural growth factor $(\mathrm{NG})^{127}$ ), a NK2-receptor agonist (saredutant ${ }^{127}$ ), and compounds which are the components of the second messenger signalling systems (inositol ${ }^{128}$ ). Thus, behavioural alterations in FSL rats are sensitive to clinically tested and putative antidepressants of different chemical classes, acting through the several neurotransmitter and/or second messenger systems in the brain. The sensitivity of the behavioural phenotype to chronic, but not acute, antidepressant treatment resembles the dynamics of the antidepressant efficacy in depressed humans, where usually weeks or months of treatment are required before the onset of clinical improvement. Therefore, it can be concluded that the normalization of behaviour in the 
FSL rats is induced by chronic treatment with different antidepressants, suggesting a high predictive validity of the FSL rat model of depression.

\section{Neurochemical Characteristics of the FSL Model of Depression}

Cholinergic Alterations in the FSL Model of Depression Apart from the basic breeding selection criteria for the development of the FSL strain, cholinergic supersensitivity, and several other alterations were found in the cholinergic system of the FSL rats. An increased uptake of radioactively labelled choline, the precursor of acetylcholine (ACh), in the cortex and higher densities of muscarinic Ach receptors in the striatum and hippocampus were found in the FSL rats. ${ }^{129}$ However, treatment with the muscarinic receptor antagonist, scopolamine, did not affect the altered behaviour in the FSL rats. ${ }^{130}$

Serotonergic Alterations in the FSL Model of Depression Extensive research has been performed on the parameters of different monoaminergic neurotransmitter systems in FSL rats. At the level of the serotonergic (5-HT) system, higher tissue levels of 5-HT and its metabolite, 5-HIAA, have been found in the prefrontal cortex, hippocampus and hypothalamus of FSL rats, relative to SPD controls. ${ }^{36}$ The tissue concentrations represent the contribution from both intra- and extracellular 5-HT, but the contribution from each compartment to the 5-HT levels measured in tissue homogenates cannot be distinguished. However, the 5-HT levels in extracellular space measured using microdyalisis were not different in the FSL rats, relative to the SPD controls, suggesting that the differences in the total tissue level of 5-HT could be attributed to the increased intracellular levels of 5-HT in FSL rats. It should be noted that the levels of 5-HT and 5-HIAA were normalized following an 18 day treatment with a $5 \mathrm{mg} / \mathrm{kg} /$ day dose of desipramine. $^{36}$

FSL rats have lower 5-HT synthesis rates throughout the brain, relative to both FRL and SPD rats, ${ }^{131}$ as measured using the $\alpha-\left[{ }^{14} \mathrm{C}\right]$ methyl-L-tryptophan ( $\alpha$-MTrp) autoradiographic method (reviewed by Diksic and Young ${ }^{132}$ ). 5-HT synthesis is normally regulated by $5-\mathrm{HT}_{1 \mathrm{~A}}$ somatodendritic autoreceptors ${ }^{133,134}$ and $5-\mathrm{HT}_{1 \mathrm{~B}}$ terminal autoreceptors. ${ }^{135-137} \mathrm{An}$ increase in extracellular 5-HT levels produced by acute treatment with the selective 5-HT reuptake inhibitor (SSRI), citalopram, ${ }^{138}$ resulted in a widespread decrease in 5-HT synthesis in FSL rats, while producing no change in SPD rats and an increase in 5-HT synthesis in FRL rats. ${ }^{139}$ On the other hand, the effect of a chronic citalopram treatment on 5-HT synthesis in FSL rats was reversed from the acute effect, while having no effect on 5-HT synthesis in the FRL strain. ${ }^{140}$ Treatment with buspirone, a $5-\mathrm{HT}_{1 \mathrm{~A}}$ re- ceptor agonist, produced largely different effects on 5-HT synthesis in the FSL and FRL rats. ${ }^{141}$ Acute treatment produces no effect on 5-HT synthesis in most regions in FSL rats, while both increases (in frontal, parietal, sensorimotor and visual cortices) and decreases (in dorsal and median raphe nuclei, amygdala, anterior olfactory nucleus, ventral hippocampus and substantia nigra - pars reticulata) of 5-HT synthesis were found in the minority of regions. On the other hand, acute buspirone induced an increase of 5-HT synthesis in the FRL rats throughout the brain, with the exception of the locus coeruleus, substantia nigra - pars reticulata and dorsal thalamus. ${ }^{141}$ Following chronic treatment with buspirone, the effect on 5-HT synthesis was reversed in both strains. In FSL rats, there was a widespread increase of 5-HT synthesis. Beside the regions showing increase in 5-HT synthesis following acute treatment, increases were now found in the amygdala, ventral thalamus, dorsal hippocampus and some striatal regions, while the decreased 5-HT following chronic treatment was limited to the median and pontine raphe nuclei. In the FRL rats, chronic buspirone treatment induced decreased 5-HT synthesis in half of the regions tested (including most of the cortical regions, nucleus accumbens, medial and lateral geniculate and dorsal raphe), but there was otherwise no change in the other half. ${ }^{141}$ These discrepant effects of the drugs acting on the 5-HT system in the FSL and FRL rats suggest a different regulation of 5-HT synthesis in those strains, which may be due to the different densities of the key receptors regulating 5-HT synthesis, such as the lower densities of $5-\mathrm{HT}_{1 \mathrm{~A}}$ and higher densities of $5-\mathrm{HT}_{1 \mathrm{~B}}$ in the $\mathrm{FSL}$ rats, ${ }^{29}$ the receptor sites serving as autoreceptors in the cell bodies and terminal regions, respectively.

The acute pharmacological inhibition by an inhibitor of tryptophan hydroxylase (TPH) activation, the key enzyme in 5-HT synthesis, did not affect the 5-HT synthesis rates in the terminal brain regions in FSL rats, unlike the effect in FRL and SPD rats. ${ }^{140}$ This suggests that the TPH in FSL rats may already be inhibited to a certain extent by the high intra-neuronal concentration of the end-product (5-HT) ${ }^{142}$ The high intra-neuronal levels of 5-HT may also account for the low 5-HT synthesis rates in the FSL rats. ${ }^{131}$ The cerebral glucose utilization (CGU), an index of neuronal activity, ${ }^{143}$ measured by $\left[{ }^{14} \mathrm{C}\right]$-2-deoxyglucose autoradiography, is shown to be higher in FSL rats, relative to both FRL and SPD rats, in most of the brain regions tested. ${ }^{140}$ This is probably due to the decreased serotonergic transmission in FSL rats, since the net effect of 5-HT action on the target neurons is a decrease in neuronal firing rate. ${ }^{144}$ This can be directly translated into lower glucose utilization, considering that glucose represents the principal energy source for the neurons and the firing rates are shown to be roughly proportional to the CGU. ${ }^{145}$ 
Dopaminergic Alterations in the FSL Model of Depression Higher tissue concentrations (3 to 8-fold) of dopamine (DA) and its metabolites, 3,4-dihydroxyphenylacetic acid (DOPAC) and homovanilic acid (HVA), have been found in the limbic regions and prefrontal cortex of FSL rats. ${ }^{36}$ This finding could be related to the higher expression levels of mRNA for tyrosinehydroxylase, ${ }^{146}$ a rate-limiting enzyme in the catecholamine synthesis pathway, in the ventral tegmental area (VTA), a region where the cell bodies of the DA neurons projecting to the nucleus accumbens (NAc) are located. This finding accords with the lower concentrations of the vesicular monoamine transporter 2 (VMAT2) in the NAc and VTA of the FSL rats. ${ }^{147}$ The function of VMAT2 is to store the newly synthesized neurotransmitters, as well as the monoamine neurotransmitters taken back into the synaptic vesicles, where they are protected from enzymatic degradation and stored until their release into the synaptic space. One of the proposed explanations of the depression-related pathophysiology in FSL rats has been the decreased release of DA in the NAc. ${ }^{122}$ DA release in the NAc is correlated with the salience of environmental stimuli. ${ }^{148}$ Neuroimaging studies in humans have suggested that NAc activation also correlates with the subjective report of pleasantness of the presented stimuli. ${ }^{149}$ Therefore, decreased DA release in the nucleus accumbens of FSL rats may be related to the anhedonia that the FSL rats exhibit under stressful conditions. ${ }^{107}$ The DA release in the NAc of FSL rats is not increased following the local application of 5-HT, unlike the effect in SPD rats. ${ }^{122}$ However, this is normalized following chronic antidepressant treatment. ${ }^{122}$ The extracellular levels of DA in the NAc are lower in the FSL rats, which are not affected by blocking the DA transporter using 1-[2-[bis(4fluorophenyl)methoxy]ethyl]-4-(3-phenylpropyl) piperazine (GBR-12909). This suggests that the lower levels are a probable consequence of lower DA release, rather than increased uptake. ${ }^{113}$

Noradrenergic Alterations in the FSL Model of Depression In parallel to the tissue levels of DA and its metabolites, 2-3 times higher tissue levels of another catecholamine neurotransmitter, norepinephrine (NA), have been found in FSL rats, relative to SPD controls, in the median raphe, NAc, hippocampus and prefrontal cortex. ${ }^{37}$ Interestingly, chronic treatment with the norepinephrine reuptake inhibitor (NRI), desipramine, normalized the tissue levels of NA concurrently with the normalization of the altered behavioural parameters in FSL rats (hypoactivity in the forced swim test). ${ }^{37}$

Other Neurotransmitter System Alterations in the FSL Model of Depression

FSL rats have lower densities of mGlu2/3 metabotropic glutamate receptors in the hippocampus, relative to FRL rats. However, in a number of other brain regions (e.g., amygdala, hypothalamus and cortical regions), these differences were not found. ${ }^{38}$ FSL rats have shown increased binding of benzodiazepines in the striatum and hippocampus, coupled with a larger behavioural effect of the GABA-ergic drugs, muscimole and diazepam. $^{148}$

Alterations of the Second Messenger Signalling Systems in the FSL Model of Depression

Differences between the FSL and FRL rats have also been found at the intracellular signalling level in the CNS. This is consistent with the neurotrophic theory of depression, ${ }^{39,139}$ which states that the depression may be a consequence of the altered second messenger signalling mechanisms (i.e., cyclic adenosine 3',5'-monophosphate; cAMP), resulting in lower levels of neurotrophic factors (such as brain-derived neurotrophic factor; BDNF). This ultimately results in lower cellularity and volume in some key brain structures implicated in the pathophysiology of depression, including the hippocampus. Consistent with the neurotrophic hypothesis of depression is the finding of decreased numbers of neurons and synapses in the hippocampus of FSL rats, which were normalized following antidepressant treatment with the NA reuptake inhibitor, imipramine. This drug also normalized the behavioural features of FSL rats. ${ }^{152}$ However, not all of the findings in the FSL rats are consistent with the neurotrophic theory. For example, levels of BDNF in the frontal cortex and hippocampus of the FSL rats were not significantly different compared to the FRL control rats. ${ }^{153}$ Indeed, chronic treatment with inositol, a precursor of the PIP signalling system component, showed the antidepressant effect in FSL rats, by reducing immobility in a forced swim test. $^{128}$ Further, chronic treatment with sildenafil, a phosphodiesterase type 5 (PDE5) inhibitor, decreased immobility in the FSL rats. ${ }^{120}$ Thus, the treatment impacting directly on one of the intracellular signalling systems appeared to be sufficient to normalize the «depressive» behaviour in FSL rats. Increased levels of arachidonic acid-containing phosphatidylcholine (AAPC) species were found in the hypothalamus and striatum of the FSL rats, and there were decreased levels of the docosahexaenoic acid (DHA)-containing species in the striatum and prefrontal cortex. ${ }^{154}$ The fact that the FSL strain, originally selected for its cholinergic supersensitivity, also shows the supersensitivity of $5-\mathrm{HT}_{1 \mathrm{~A}}$ receptors (as measured by the hypothermic effects following the acute injection of the selective $5-\mathrm{HT}_{1 \mathrm{~A}}$ agonist, buspirone and 8-OH-DPAT), ${ }^{155}$ further suggests that the FSL rats may differ from normal rats at the intracellular signalling level, and are thereby capable of affecting the multiple neurotransmitter systems functions. 


\section{Depression as a Result of Gene-Environment Inter-} action

Building on the gene-environment theory of depression, which posits that the development of depression is a result of the complex interplay between genetic predisposition and environmental adversities, ${ }^{156,157}$ a recent line of research has exploited the consequences of the well established stress-inducing paradigm, maternal separation (MS), on the FSL rats and FRL controls. In the MS paradigm, pups are separated from the dams for between 15 minutes and 6 hours daily, during the first two weeks of life. ${ }^{158}$ In normal rats, this results in the development of neurochemical and behavioural changes expressed even in adulthood, such as increased anxiety and stress-reactivity (reviewed by Holmes et al. ${ }^{159}$ ). It was presumed that exposing the rats with a genetic predisposition for the development of depressive phenotype, such as FSL, to maternal separation, would model the gene-environment scenario in the development of depression in susceptible humans. The study using maternal separation in FSL and FRL pups has found differences in the protein expression in several regions implicated in the pathophysiology of depression in humans, including the frontal cortex and hippocampus. ${ }^{160}$ Moreover, chronic treatment with the SSRI, escitalopram, has decreased immobility on the forced swim test in the non-MS FSL rats, but had no effect on this behavioural parameter in the MS FSL rats, suggesting that the cumulative effect of the genetic predisposition to depression and early life adversity may result in an altered behavioural phenotype resistant to antidepressant treatment. ${ }^{161}$ Differences were also found between the FSL and FRL rats in the serum levels of proteins involved in lipid metabolism and immunity. ${ }^{162}$

Although the straight-forward interpretation of these findings is difficult, it appears that stressful conditions during the early developmental phase have different effects on brain development, as well as the behavioural phenotype, at the adult stage, in the FSL rats and the normal controls.

\section{CONCLUSION}

Inherent weakness of rodent models of most psychiatric disorders, especially mood disorders, is that some of the core features of these disorders (e.g., excessive feelings of guilt or suicidal ideation, in the case of depression) are too complex to be induced in rodents and also could not be reliably assessed in any animal model due to their introspective character. However, OBX and FSL models exhibit their validity in two primary ways: 1) the physiological and behavioural alterations characterizing these models are normalized by the chronic, but not acute, treatment with clinically active antidepressants targeting various neurochemical systems; and 2) the same alterations are not changed following treatment with psychotropic drugs other than antidepressants. This makes the FSL and OBX models useful tools for drug candidate screening in the pharmaceutical industry. Nevertheless, concerns have been raised that relying on existing models as screening tools could result in falsenegative findings when testing the compounds with the mechanisms of action different from the present antidepressants.

Further, it can be stated that there is a great need for the study of depression and its treatment in good animal models of depression, rather than studying treatments in normal rats, as one can then use a homogeneous population group and any conclusions drawn can more likely be correlated to some human depression. The data summarized also suggest that studies of brain regional 5-HT synthesis, as one of the most important presynaptic processes, are very important for a better understanding of depression itself and neurochemical changes produced by treatments.

Acknowledgements: The research reported here was supported in part by grants from the Croatian Ministry of Science, Education and Sports (219-1081970-2032). T.K. was also supported by a grant from The National Foundation for Science, Higher Education and Technological Development of the Republic of Croatia. We would also like to thank Ms. Valerie-Ann Cherneski for her editorial help.

\section{REFERENCES}

1. L.L. Judd, Int. Clin. Psychopharmacol, Suppl 4 (1995) 5-10. Erratum in: Int. Clin. Psychopharmacol 11 (2) (1996) 153.

2. R.C. Kessler, P. Berglund, O. Demler, R. Jin, K.R. Merikangas and E.E. Walters, Arch. Gen. Psychiatry 62 (2005) 593-602. Erratum in: Arch. Gen. Psychiatry 62 (2005) 768.

3. DMS-IV, The Diagnostic Statistical Manual of Mental Disorders-IV (1994)

4. G. Yadid and A. Friedman, Prog. Brain Res. 172 (2008) 265-86.

5. W.F. Byerley and S.C. Risch, J. Clin. Psychopharmacol. 5 (1985) 191-206.

6. C.A. Naranjo, L.K. Tremblay and U.E. Busto, Prog. Neuropsychopharmacol. Biol. Psychiatry 25 (4) (2001) 781-823.

7. E.J. Nestler, M. Barrot, R.J. DiLeone, A.J. Eisch, S.J. Gold and L.M. Monteggia, Neuron 34 (2002) 13-25.

8. I.D. Neumann, G. Wegener, J.R. Homberg, H. Cohen, D.A. Slattery, J. Zohar, J.D. Olivier and A.A. Mathé, Prog Neuropsychopharmacol Biol Psychiatry (2011),

9. A.B. Fernando and T.W. Robbins, Annu Rev Clin Psychol 7 (2011) 15.1-15.23

10. J.P. Kelly, A.S. Wrynn and B.E. Leonard, Pharmacol. Ther. 74 (1997) 299-316.

11. M.F. O’Neil and N.A. Moore, Human Psychopharmacol. Clin. Exp. 18 (2003) 239-254.

12. J.F. Cryan, A. Markou and I. Lucki, TRENDS in Pharmacol. Sciences 23 (2002) 238-245.

13. P. Willner and P.J. Mitchell, Behav. Pharmacol. 13(2002) 169-188. 
14. G.M. Shepherd, Physiological. Reviews. 52 (1972) 864-917.

15. 15. J.D. Jentsch and J.R. Taylor, Psychopharmacology 146 (1999) 373-390.

16. E. Dremencov M.E. Newman, N. Kinor G. Blatman-Jan, C.J. Schindler, D.H. Overstreet and G. Yadid, Neuropharmacol. 48 (2005) 34-42.

17. E. Dremencov, I. Gispan-Herman, M. Rosenstein, A. Mendelman, D.H. Overstreet, J. Zohar and G. Yadid, Prog. Neuropsychopharmacol. Biol. Psychiatry 28 (2004) 141-147.

18. A. Mar, E. Spreekmeester and J Rochford, Psychopharmacology (Berl) 150 (2000) 52-60.

19. D.H. Overstreet and R.W. Russell, Psychopharmacol (Berl) $\mathbf{7 8}$ (1982) 150-155.

20. D.H. Overstreet, R.W. Russell, S.C. Helps and M. Messenger, Psychopharmacology (Berl) 65 (1979) 15-20.

21. J.A. Jesberger and J.S. Richardson, Int. J. Neuroscience 38 (1988) 241-265.

22. A.R. Lumia, M.H. Teicher, F. Salchli, E. Ayers and B. Possidente, Brain Res. 587 (1992) 181-185.

23. D.H. Overstreet, E. Friedman, A.A. Mathé and G.Yadid, Neuroscien. Biobehav. Rev 29 (2005) 739-759.

24. C.Song and B.E. Leonard, Neuroscien. Biobehav. Rev. 29 (2005) 627-647.

25. N. Nelson, J. Physiology (Paris) 87(1993) 171-178.

26. P. Blier, Eur. Neuropsychopharmacol. 13 (2003) 57-66.

27. H. Sato, I. Skelin, G. Debonnel and M. Diksic, Brain Res. Bull. 75 (2008) 545-55.

28. H. Sato, I. Skelin and M. Diksic, Brain Res. 1345 (2010) 28-44.

29. K. Nishi, K. Kanemaru and M. Diksic, Neurochem. Int. 54 (2009) 299-307.

30. M.Yates, A. Leake, J.M. Cy, A.F. Fairbairn, I.G. McKeith and I.N. Ferrier, Biol. Psychiatry 27(5) (1990) 489-496.

31. J.J. Mann, M.D. Underwood and V. Arango, In: Biology of schizophrenia and affective disease, Watson SJ (Ed). American Psychiatric Press, Washington, 1996, pp 197-221.

32. B. Earley, M. Glennon, M. Lally, B.E. Leonard and J.L. Junien, Human Psychopharmacol. 9 (1994) 397-407.

33. J.H. Meyer, S. Kapur, B. Eisfeld, G.M. Brown, S. Houle, J. DaSilva, A.A. Wilson, S. Rafi-Tari, H.S. Mayberg and S.H. Kennedy, Am. J. Psych. 158 (2001) 78-85.

34. L.N. Yatham, P.F. Liddle, J.Dennie, I.S. Shiah, M.J. Adam, C.J. Lane, R.W. Lam and T.J. Ruth, Arch. Gen. Psychiatry 56 (1999) 705-711.

35. D.H. Overstreet and G. Griebel, Eur. J. Pharmacol. 497 (2004) 49-53.

36. A.Zangen, D.H. Overstreet and G. Yadid J. Neurochem. 69 (1997) 2477-2483.

37. A. Zangen, D.H. Overstreet and G. Yadid (1999) Brain Res. 824 243-250.

38. F. Matrisciano, A. Caruso, R. Orlo, M. Marchiafava, V. Bruno, G. Battaglia, S.H. Gruber, D. Melchiorri, R. Tatarelli, P. Girardi, A.A. Mathe and F. Nicoletti, Neuropharmacology 55 (2008) 525-531.

39. R.S. Duman, G.R. Heninger and E.J. Nestler, Arch. Gen. Psychiatry 54 (1997) 597-606

40. A.Watanabe, Y. Tohyama, K.Q. Nguyen, S. Hasegawa, G. Debonnel and M. Diksic, J. Neurochem. 85 (2003) 469-475.

41. S. Hasegawa, A.Watanabe, K.Q. Nguyen, G. Debonnel and Diksic, M. Psychopharmacol. (Berl). 179 (2005) 781-790.

42. H. van Riezen, H. Schnieden and A. F Wren, Br. J. Pharmacol. 60 (1977) 521-528.

43. J. Vetulani, F. Sulser, Nature 257 (1975) 495-496.

44. C. Song, B. Earley and B. E. Leonard, Brain Behav. Immun. 10 (1996) 1-16.

45. M. Cattarelli and A. Demael, Experientia 42 (2) (1986) 169-71.

46. D. Zhou, G. Grecksch, A. Becker, C. Frank, J. Pilz and G. Huether, J. Neurosci. Res. 54 (1998) 109-116.
47. B. Robinzon, N. Snapir and M. Perek, Brain Res. Bull. 2 (1977) 263-271.

48. C. H. Vinkers, M. E. Breuer, K. G. Westphal, S. M. Korte, R. S Oosting, B. Olivier and L. Groenink, Neuroscience 159 (2009) (1) 39-46.

49. H. van Riezen and B. E. Leonard, Pharmacol. Ther. 47 (1990) 21-34.

50. H.M. Kang, J. Jin, S. Lee, J. Ryu and C. Park, Neuroreport 21 (3) (2010) 179-184.

51. U. Dannlowski, P. Ohrmann, C. Konrad, K. Domschke, J. Bauer, H. Kugel, C. Hohoff, S. Schoning, A. Kersting, B.T. Baune, L.S. Mortensen, V. Arolt, P. Zwitserlood, J. Deckert, W. Heindel and T. Suslow, Int. J. Neuropsychopharmacol. 12 (2009) 11-22.

52. A. S. Wrynn, C. P. Mac Sweeney, F. Franconi, L. Lemaire, D. Pouliquen, S. Herlidou, B. E. Leonard, J. Gonde and J. D. Certaines, Brain Res. 879 (2000) 193-199.

53. K. Jaako-Movits, T. Zharkovsky, M. Pedersen and A. Zharkovsky, Cell Mol. Neurobiol. 26 (2006) 1559-1570.

54. C.H. Kellner, D.R. Rubinow and R.M. Post, J. Affect. Disord. 10 (1986) 215-219.

55. A. Saitoh, K. Yamaguchi, Y. Tatsumi, H. Murasawa, A. Nakatani, N. Hirose, M. Yamada, Yamada and J. Kamei, Psychopharmacology (Berl) 191 (2007) 857-865.

56. D. Klein and T.S. Brown, J. Comp. Physiol. Psychol. 68 (1969) 107-110.

57. S. Hozumi, O. Nakagawasai, K. Tan-No, F. Niijima, F. Yamadera, A. Murata, Y. Arai, H. Yasuhara, and T. Tadano, Behav. Brain Res. 138 (2003) 9-15.

58. M. Diksic, S. Nagahiro and T.L. Sourkes, J. Neural. Transm., Suppl 29 (1990) 131-140.

59. M. Diksic, S. Nagahiro, T.L. Sourkes and Y.L. Yamamoto, $J$ Cereb. Blood Flow Metab. 10 (1990) 1-12.

60. I.V. Nesterova, E.V. Gurevich, V.I. Nesterov, N.A. Otmakhova and N.V. Bobkova, Prog. Neuropsychopharmacol. Biol. Psychiatry 21 (1997) 127-140.

61. K.Q. Nguyen, Y. Tohyama, A. Watanabe, S. Hasegawa, I. Skelin and M. Diksic, Neurochem. Int. 54 (2009) 161-171.

62. G. Grecksch, D. Zhou, C. Franke, U. Schroder, B. Sabel, A. Becker and G. Huether, Br. J. Pharmacol. 122 (1997) 1725-1731.

63. K. Kato, T. Awasaki, K. Ito, Development 136 (2009) 51-59.

64. M. Kadekaro, W.H. Vance, M.L. Terrell, H. Gary, Jr., H.M. Eisenberg and L. Sokoloff, Proc. Natl. Acad. Sci. U S A 84 (1987) 5492-5495.

65. I. Skelin, H. Sato and M. Diksic, Brain Res. Bull. 76 (2008) 485-492.

66. H. M. van der Stelt, , M. E. Breuer B. Olivier and H. G. Westenberg, Biol. Psychiatry 57 (2005) 1061-1067.

67. I. Skelin, H. Sato, T. Kovačević and M. Diksic, Psychopharmacol. (Berl) (2009) 207 (2) 315-323.

68. I. Skelin, H. Sato and M. Diksic, Brain Res. 1310 (2010) $162-171$.

69. 69. A.C. Shakesby, R. Anwyl and M.J. Rowan, J. Neurosci. 22 (2002) 3638-3644.

70. A. Watanabe, S. Hasegawa, K. Nishi, K. Q. Nguyen and M. Diksic, Brain Res. Bull. 69 (2006), (2), 101-108.

71. D. Healy, P.A. Carney and B.E. Leonard, J. Psychiatr. Res. 17 (1982) 251-260

72. C.V. Masini, P.V. Holmes, K.G. Freeman, A.C. Maki and G.L. Edwards, Physil. Behav. 81 (2004)111-119.

73. R.B. Lingham and Z. Gottesfeld, J. Neurosci. 6 (1986) 2208-2214.

74. G.G. Nomikos, A.P. Zis, G. Damsma, H.C. Fibiger, Psychopharmacology 105 (1991) 230-238.

75. A.S. Brown and S. Gershon, J Neural Transm Gen. Sect. 91 (1993) 75-109.

76. B.J. Ciliax, C. Heilman, L.L. Demchyshyn, Z.B. Pristupa, E. 
Ince, S.M. Hersch, H.B. Niznik and A.I. Levey, J. Neurosci. 15 (1995) 1714-1723.

77. P.L. Mc Geer, J.C. Eccles, E.G. Mc Geer, Molecular neurobiology of the mammalian brain. Plenum Press, 1987, New York.

78. D.T. Monaghan, R.J. Bridges and C.W. Cotman, Ann. Rev. Pharmacol. Toxicol. 29 (1989) 365-402.

79. J.M. Wierońska, B. Legutko, D. Dudys and A. Pilc, Pharmacol. Rep. 60 (2008) 844-855.

80. Y.-J. Ho, Y.-C. Chang, T.-M. Liu, M.-Y. Tai, C.-S. Wong, Y.-F. Tsai, Neuroscience Letters 287 (2000) 11-120.

81. M.T. Lowy, L. Gault, and B.K. Yamamoto, J. Neurochem. 61 (1993) 1957-1960

82. K.D. Cairncross, A. Wren, B. Cox, and H. Schnieden, Physiol. Behav. 19 (1977) 485-487.

83. J. M. Wieronska, B. Szewczyk, P. Branski, A. Palucha and A. Pilc, Amino Acids 23 (2002) 213-216.

84. A. Palucha, P. Branski, B. Szewczyk, J. M. Wieronska, K. Klak and A. Pilc, Pharmacol. Biochem. Behav. 81 (2005) 901-906.

85. K. Takahashi, H. Murasawa, K. Yamaguchi, M. Yamada, A. Nakatani, M. Yoshida, T. Iwai, M. Inagaki and A. Saitoh, Behav. Brain Res. 216 (2011) 46-52.

86. Y. Ho, T. Liu, M. Tai, Z. Wen, R.S. Chow, Y. Tsai and C. Wong, Brain Res. 900 (2001) 214-218.

87. M. Robichaud, V. Beauchemin, N. Lavoie, T. Dennis and G. Debonnel, Synapse 42 (2001) 95-103.

88. T. Tadano, S. Hozumi, F. Yamadera, A. Murata, F. Niijima, K. Tan-No, O. Nakagawasai and K. Kisara, Methods Find. Exp. Clin. Pharmacol. 26 (2004) 93-97.

89. H.Araki, K. Suemaru and Y. Gomita, Jpn. J. Pharmacol. 88 (2002) 133-138.

90. H. Yoshimura, Y. Gomita and S. Ueki, Pharmacol. Biochem. Behav. 2 (1974) 703-705.

91. F. Han, N. Shioda, S. Moriguchi, Y. Yamamoto, A.Y. Raie, Y. Yamaguchi, M. Hino, K and Fukunaga, J. Pharmacol. Exp. Ther. 326 (2008) 127-134.

92. A. Nakajima, T. Yamakuni, M. Haraguchi, N. Omae, S.-Y. Song, C. Kato, O. Nakagawasai, T. Tadano, A. Yokosuka, Y. Mimaki, Y. Sashida and Y. Ohizumi, J. Pharmacol. Sci. 105 (2007) 122-126.

93. S.D. Norrholm and C.C. Ouimet. Synapse 42 (2001) 151-163.

94. M.H. Millan, S. Patel and B.S. Meldrum, Brain Res. 398 (1986) 204-206.

95. M. Muramatsu, J. Tamaki-Ohashi, C. Usuki, H. Araki and H. Aihara, Neuropharmacol. 27 (1988) 603-609.

96. I.Y. Aleksandrova, V.V. Kuvichkin, I.A. Kashparov, N.I. Medvinskaya, I.V. Nesterova, S.M. Lunin, A.N. Samokhin and N.V. Bobkova, Biochemistry (Mosc) 69 (2004) 176-180.

97. M. Heilig and E. Widerlöv, Acta Psychiatr. Scand. 82 (1990) $95-114$.

98. N. J. Rutkoski, A. A. Lerant, C. M. Nolte, J. Westberry and C. W. Levenson, Brain Res. (2002) 951 (1), 69-76.

99. I. Tasset, R. Drucker-Colin, J. Pena, I. Jimena, P. Montilla, F. J. Medina and I. Tunez, Neurochem. Res. 35 (2010) 1182-1187.

100. A. Zafir and N. Banu, Eur. J. Pharmacol. 572 (2007) (1), 23-31.

101. F. Karege, G. Vaudan, M. Schwald, N. Perroud and R. La Harpe, Brain Res. Mol. Brain Res. 136 (2005) 29-37.

102. K.R. Luo, C.J. Hong, Y.J. Liou, S.J. Hou, Y.H. Huang and S.J. Tsai, Prog. Neuropsychopharmacol. Biol. Psychiatry 34 (2010) 1433-1439.

103. B. Chen, D. Dowlatshahi, G.M. MacQueen, J.F. Wang and L.T. Young, Biol. Psychiatry 50 (2001) 260-265.

104. P. Vieyra-Reyes, Y. S. Mineur, M. R. Picciotto, I. Tunez, R. Vidaltamayo and R. Drucker-Colin, Brain Res Bull 77 (2008) $13-18$.

105. A. Saitoh, M. Yamada, K. Takahashi, K. Yamaguchi, H. Murasawa, A. Nakatani, Y. Tatsumi, N.Hirose and J. Kamei, Brain Res 1208 (2008) 160-169.
106. D.S. Janowsky, C. Risch, D. Parker, L. Huey and L, Judd, Psychopharmacol. Bull. 16 (1980) 29-31.

107. O. Pucilowski and D.H. Overstreet, Brain Res.Bull. 32 (1993) 471-475.

108. R.D. Porsolt, P.M. Le and M. Jalfre, Nature 266 (1977) 730-732.

109. L. Prut and C. Belzung, Eur. J. Pharmacol. 463 (2003) 3-33.

110. G.D. Schiller, L.C. Daws, D.H. Overstreet and J. Orbach, Brain Res. Bull. 26 (1991) 433-435.

111. P. Willner, R. Muscat and M. Papp, Clin. Neuropharmacol. 15 (1992) Suppl 1 Pt A: 550A-551A.

112. K. Matthews, B.A. Baldo, A. Markou, O. Lown, D.H. Overstreet and G.F. Koob, Physiol. Behav. 59 (1996) 1155-1162.

113. I. Roth-Deri, A. Friedman, L. Abraham, E.Lax, Y. Flaumenhaft, Y. Dikshtein and G. Yadid, Eur. J. Neurosci. 30 (2009) 485-492.

114. M.P. Austin, P. Mitchell and G.M. Goodwin, Br. J. Psychiatry 178 (2001) 200-206.

115. R.M. Benca, D.E. Overstreet, M.A. Gillil, D. Russell, B.M. Bergmann and W.H. Obermeyer, Neuropsychopharmacol. 15 (1996) 45-51.

116. P.J. Shiromani, J.C. Gillin and S.J. Henriksen, Annu. Rev. Pharmacol. Toxicol. 27 (1987) 137-156.

117. J. Adrien, Sleep Med. Rev. 6 (2002) 341-351.

118. E.M. Friedman, K.A. Becker, D.H. Overstreet and D.A. Lawrence, Psychosom. Med. 64 (2002) 267-273.

119. P. Willner, Prog. Neuropsychopharmacol. Biol. Psychiatry, 10 (1986) 677-690.

120. N. Liebenberg, B.H. Harvey, L. Brand and C.B. Brink, Behav. Pharmacol. 21 (2010) 540-547.

121. N. Kokras, K. Antoniou, C. Dalla, S. Bekris, M. Xagoraris, D.H. Ovestreet and Z. Papadopoulou-Daifoti, J. Psychopharmacol. 23 (2009) 945-956.

122. A. Zangen, R. Nakash, D.H. Overstreet and G. Yadid, Psychopharmacol. (Berl) 155 (2001) 434-439.

123. Y. Tizabi D.H. Overstreet A.H. Rezvani V.A. Louis, E. Jr. Clark, D.S. Janowsky and M.A. Kling, Psychopharmacol (Berl) 142 (1999) 193-199.

124. D.H. Overstreet, J. Stemmelin and G. Griebel, Pharmacol. Biochem Behav. 89 (2008) 623-626.

125. D.H. Overstreet, O. Pucilowski, M.C. Retton, P. Delagrange and B. Guardiola-Lemaitre, Neuroreport 9 (1998) 249-253.

126. D.H. Overstreet, A. Keeney and S. Hogg, Eur. J. Pharmacol. 492 (2004) 195-201.

127. D.H. Overstreet, V.M. Naimoli and G. Griebel, Pharmacol. Biochem. Behav. 96 (2010) 206-210.

128. H. Einat, R.H. Belmaker, A. Zangen, D.H. Overstreet and G. Yadid, Depress. Anxiety. 15 (2002) 148-151.

129. D.H. Overstreet, R.W. Russell, A.D. Crocker and G.D. Schiller, Brain Res. 294 (1984) 327-332.

130. D.H. Overstreet, O Pucilowski, A.H. Rezvani and D.S. Janowsky, Psychopharmacol. (Berl) 121 (1995) 27-37.

131. S. Hasegawa, K. Nishi, A.Watanabe, D.H. Overstreet and M. Diksic, Neurochem. Int. 48 (2006) 358-366.

132. M. Diksic and S.N. Young, J. Neurochem. 78 (2001) 1185-1200.

133. H. Okazawa, F.Yamane, P. Blier and M. Diksic, J. Neurochem. 72 (1999) 2022-2031.

134. Y. Tohyama, F. Yamane, M.F. Merid and M. Diksic Europ Neuropsychopharmacol 11 (2001) 193-202.

135. Y. Tohyama, F.Yamane, M.M. Fikre, P. Blier and M. Diksic, $J$. Neurochem. 80 (2002) 788-798.

136. S. Hasegawa, A. Watanabe, K. Nishi, K.Q. Nguyen and M. Diksic, Neurochem. Int. 46 (2005) 261-272.

137. C.F. Dobson, Y. Tohyama, M. Diksic and E. Hamel, Cephalalgia 24 (2004) 2-11.

138. C. Moret, M. Briley, Naunyn Schmiedebergs Arch Pharmacol. 351 (1995) 377-384.

139. K. Kanemaru, S. Hasegawa, K. Nishi and M. Diksic, Brain Res. Bull. 77 (2008) 214-220. 
140. K. Kanemaru, K. Nishi and M. Diksic Neurochem. Int. 55 (2009) 529-535.

141. K. Nishi, K. Kanemaru, S. Hasegawa, A. Watanabe and M. Diksic, Neurochem. Int. 54 (2009) 205-214.

142. J.B. Macon, L. Sokoloff and J. Glowinski, J. Neurochem. 18 (1971) 323-331.

143. L. Sokoloff, Brain 102 (1979) 653-668.

144. J.J. Grome and A.M. Harper J. Neurochem. 46 (1986) 117-124.

145. P.Yarowsky, M. Kadekaro and L. Sokoloff Proc. Natl. Acad. Sci. U.S.A 80 (1983) 4179-4183.

146. L. Serova, E.L. Sabban, A. Zangen, D.H. Overstreet and G. Yadid, Brain Res. Mol. Brain Res. 63 (1998) 133-138.

147. K. Schwartz, G.Yadid, A. Weizman and M. Rehavi, Brain Res. 965 (2003) 174-179.

148. M.F. Roitman, R.A. Wheeler, R.M. Wightman and R.M. Carelli, Nat. Neurosci. 11 (2008) 1376-1377.

149. D. Sabatinelli, M.M. Bradley, P.J. Lang, V.D. Costa and F. Versace J. Neurophysiol. 98 (2007) 1374-1379.

150. S. Pepe, D.H. Overstreet and A.D. Crocker, Pharmacol. Biochem. Behav. 31 (1988) 15-19.

151. H.K. Manji Quiroz J. Sporn J.L. Payne K. Denicoff A. Gray, C.A. Zarate Jr. and D.S. Charney, Biol. Psychiatry 53 (2003) 707-742.

152. F. Chen, T.M. Madsen, G.Wegener and J.R. Nyengaard, Hippocampus 20 (2009) 1376-1384.

153. B. Elfving, P.H. Plougmann, H.K. Muller, A.A. Mathe, R.
Rosenberg and G. Wegener, Int. J. Neuropsychopharmacol. 13 (2010) 563-572.

154. P. Green, N. Anyakoha, G. Yadid, I. Gispan-Herman and A Nicolaou, Prostaglins Leukot. Essent. Fatty Acids 80 (2009) 213-220.

155. D.H. Overstreet, A.H. Rezvani and D.S. Janowsky , Biol. Psychiatry 31 (9) (1992) 919-936.

156. T.C. Eley, K. Sugden, A. Corsico, A.M. Gregory, P. Sham, P. McGuffin, R. Plomin andI.W. Craig, Mol. Psych. 9 (2004) 908-915.

157. R.L. Sjöberg, K.W. Nilsson, N. Nordquist, J. Ohrvik, J. Leppert, L. Lindström and L. Orel, Int. J. Neuropsychopharmacol. 9 (2006) 443-449.

158. M.C. Moffett, A. Vicentic, M. Kozel, P. Plotsky, D.D. Francis and M.J. Kuhar, Biochem. Pharmacol. 73 (2007) 321-30.

159. A. Holmes, A.M. le Guisquet, E. Vogel, R.A. Millstein, S. Leman and C. Belzung, Neurosci. Biobehav. Rev. 29 (2005) 1335-1346.

160. C. Piubelli, M. Vighini, A.A. Mathé, E. Domenici and L. Carboni, Int. J. Neuropsychopharmacol. (2010) 1-38;

161. C. Piubelli, M. Vighini, A.A. Mathé, E. Domenici and L. Carboni, Int. J. Neuropsychopharmacol. (2010) 1-22;

162. L. Carboni, S. Becchi, C. Piubelli, A. Mallei, R. Giambelli, M. Razzoli, A.A. Mathé, M. Popoli and E. Domenici, Prog. Neuropsychopharmacol. Biol. Psychiatry 34 (2010) 1037-1048. 